\title{
EFFEKTE DER SKALP-AKUPUNKTUR AUF DIE HERZRATEN- VARIABILITÄT BEI PATIENTEN MIT SCHLAFSTÖRUNGEN
}

\author{
Ein Projektbericht (Studiendesign) zur Teleakupunktur mit Harbin in COVID-19-Pandemie-Zeiten
}

\section{Effects of Scalp Acupuncture on Heart Rate Variability in Patients with Insomnia A Project Report (Study Design) on Tele-Acupuncture with Harbin in COVID-19-Pandemic-Times}

\section{Zusammenfassung}

Dieses Forschungsprojekt im Bereich der High-TechAkupunktur befasst sich im Rahmen einer erfolgreichen chinesisch-österreichischen Zusammenarbeit mit klinischen Messungen der Variabilität der Herzfrequenz bei transkontinentaler Skalp-Akupunktur an Freiwilligen und PatientInnen mit Schlaflosigkeit. Registrierungen der Herzrate (HR) und der Herzratenvariabilität (HRV) sollen unter standardisierten Bedingungen in Harbin, China, durchgeführt werden. Die Datenanalysen dazu werden in Graz realisiert. Das Projekt ist als randomisierte klinische Studie mit zwei PatientInnengruppen konzipiert. Jede Gruppe besteht aus 20 PatientInnen. In der Behandlungsgruppe werden die Akupunkturpunkte LG 20 (Baihui), LG 24 (Shenting) und Gb 13 (Benshen; bilateral) sowie einige allgemeine Körperpunkte einschließlich EX-HN 3 (Yintang), EX 8 (Anmian I), KS 6 (Neiguan; bilateral), He 7 (Shenmen; bilateral), Bl 62 (Shenmai; bilateral) und Ni 6 (Zhaohai; bilateral) für die Behandlung von Schlaflosigkeit verwendet. Die Kontrollgruppe erhält nur allgemeine Körper-Akupunktur. Zusammen mit den Ergebnissen frühererTele-Akupunkturstudien können die vorliegenden Ergebnisse als Grundlage für die Weiterentwicklung von SkalpAkupunkturstimulationsverfahren im Sinne von ergänzenden Anwendungen bei der Behandlung und/oder Verringerung von Schlaflosigkeit dienen. Beide Institutionen haben gute ExpertInnen für diese Art von Experimenten.

\section{Schlüsselwörter}

Akupunktur, Skalp-Akupunktur, Herzratenvariabilität, evidenzbasierte Komplementärmedizin

\section{Summary}

This project within a successful Sino-Austrian cooperation on high-tech acupuncture deals with clinical transcontinental scalp acupuncture related to heart rate variability measurements in volunteers and patients with insomnia. Heart rate (HR) and heart rate variability (HRV) registrations should be performed under standardized conditions in Harbin, China. The data analyses should be performed in Graz,Austria. The project is designed as a randomized clinical trial, with two groups of patients; each group consists of 20 patients. In the treatment group we will use scalp acupuncture points DU 20 (Baihui), DU 24 (Shenting), and GB 13 (Benshen; bilateral) and also some general body points for insomnia treatment including EX 1 (Yintang), EX 8 (Anmian I), PC 6 (Neiguan; bilateral), HT 7 (Shenmen; bilateral), UB 62 (Shenmai; bilateral), and KI 6 (Zhaohai; bilateral).The control group will only receive general body acupuncture. Similar to previous preliminary measurements, the electrocardiograms should be recorded in China by instruments from China and also by an HRV Medilog AR12 system (from TCM Research Center, Graz, Austria) during scalp acupuncture and also during scalp and body acupuncture respectively. Together with the results of previous tele-acupuncture studies, the present results can serve as a basis for further development of scalp-acupuncture stimulation procedures for complementary use in treating and/or reducing insomnia. Both institutions have good experts for this kind of experiments.

\section{Keywords}

acupuncture, scalp-acupuncture, heart rate variability, evidence-based complementary medicine 


\section{Einleitung}

\section{Zusammenarbeit zwischen der Medizinischen Universität Graz und der Heilongjiang-Univer- sität für TCM in Harbin}

Bereits seit mehr als 12 Jahren arbeitet das Forschungszentrum für Traditionelle Chinesische Medizin (TCM) unter der Leitung von Univ.-Prof. DDr. Gerhard Litscher an der Medizinischen Universität Graz höchst erfolgreich mit internationalen Partnern zusammen. Einer dieser wissenschaftlichen Kooperationspartner, die Heilongjiang-Universität für TCM, wurde 1954 gegründet und entwickelte sich rasch zu einer der bekanntesten TCM-Universitäten in China (Abb. 1).

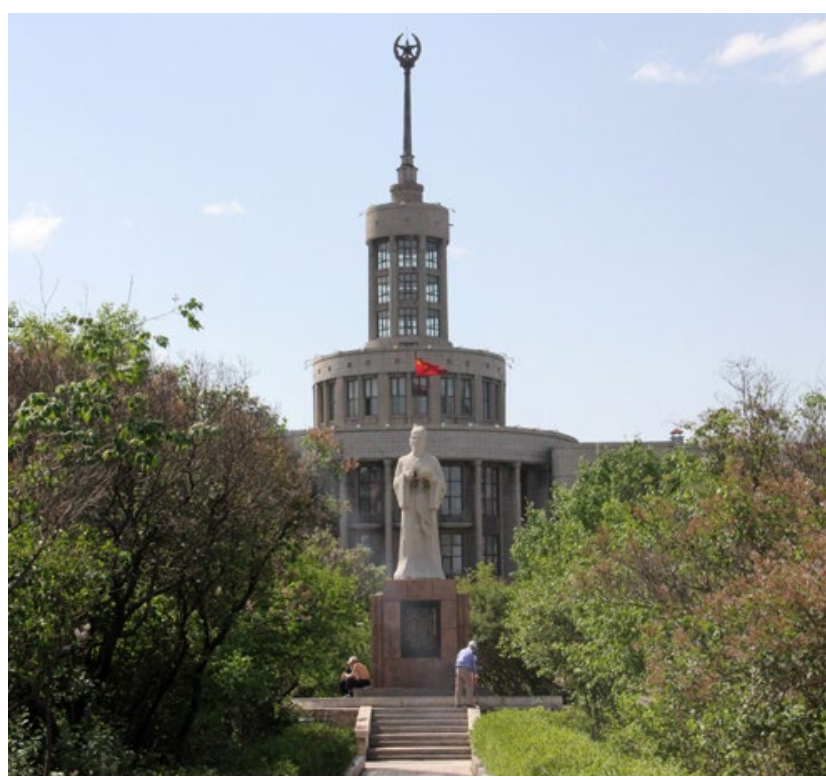

Abb. 1: Heilongjiang-Universität für Traditionelle Chinesische Medizin in Harbin, China

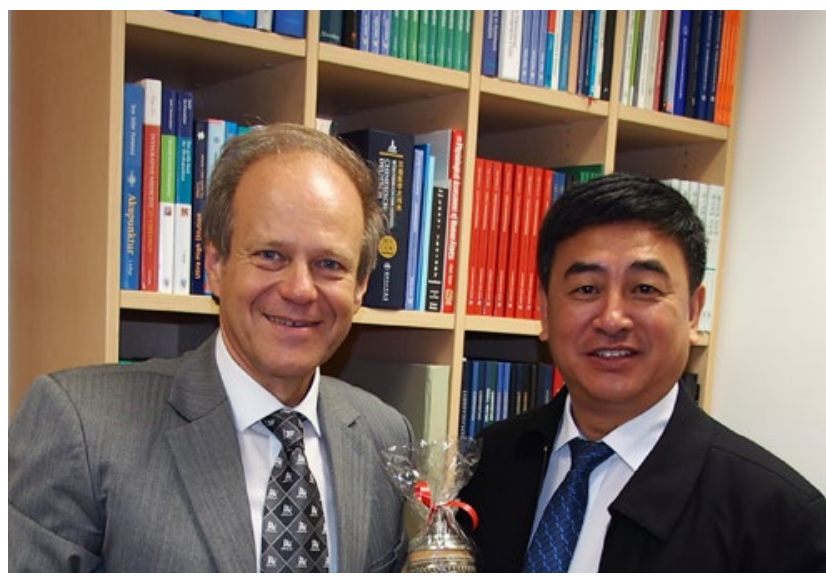

Abb. 2: Prof. Zhongren Sun, derzeitiger Präsident der HeilongjiangUniversität für Chinesische Medizin, Harbin und Prof. Gerhard Litscher an der Medizinischen Universität in Graz noch vor der Corona-Krise
Nach mehr als einem halben Jahrhundert Bautätigkeiten hat sich die Heilongjiang-Universität mittlerweile zu einer der TCM-Universitäten mit einem hohen Niveau an Lehre, Forschung und Gesundheitswesen im In- und Ausland entwickelt (Top 6 unter allen Universitäten für Chinesische Medizin). Die Universität befindet sich in der nördlichen Stadt Harbin in China. Sie besteht aus 11 Hochschulen, 11 angegliederten Krankenhäusern, einem Forschungsinstitut, 27 Lehrkrankenhäusern und 70 Praxisinstitutionen. In 34 Fächern kann eine Promotion verliehen werden, 41 Fächer haben einen Master-Abschluss. Die Universität deckt mehrere Disziplinen ab: Medizin, Wissenschaft, Kultur, Ingenieurwesen usw. Im Vorjahr gab es 15.221 Vollzeitstudenten, darunter 299 Doktoranden, 1.947 Masterstudenten, 1.742 Junior-College-Studenten und 288 ausländische Studenten.

Akupunktur ist eine der wichtigsten Forschungsdisziplinen dieser renommierten Universität. Die Medizinische Universität Graz kooperiert mit der Heilongjiang-Universität für Chinesische Medizin zum Thema High-TechAkupunkturforschung. In den letzten Jahren wurden 10 gemeinsame Veröffentlichungen, die meisten davon in Top-20\%-Zeitschriften, publiziert.

Prof. Sun Zhongren, der gegenwärtige Präsident dieser Universität, fördert die Zusammenarbeit zwischen Graz und Harbin und wurde von seinen Kollegen Prof. Tang Qiang und Prof. Zhu Luwen noch vor der Corona-Krise bei einem Besuch am TCM-Forschungszentrum der MedUni Graz begleitet (Abb. 2).

Für die Zukunft einigte man sich bei den Gesprächen in Harbin und Graz darauf, die bestehende Zusammenarbeit $\mathrm{zu}$ intensivieren und weitere gemeinsame Projekte voranzutreiben.

Nun, ausgerechnet während der COVID-19-Pandemie, wurde seitens des OEAD und des Österreichischen Bundesministeriums für Bildung, Wissenschaft und Forschung ein von G. Litscher eingereichtes Projekt für die weitere Zusammenarbeit mit dem im Titel stehenden gleichlautenden Projektnamen genehmigt (Dauer: 1.7.2020 bis vorläufig 28.2.2022). Bestimmt spielte bei der Entscheidungsfindung und bei den Gutachtern des Ministeriums auch die Tatsache, dass es sich dabei um eine im weitesten Sinne „Tele-Akupunkturstudie“ in der Corona-Zeit handelt, eine nicht unerhebliche Rolle. Ähnliche Projekte konnten ja bereits erfolgreich beendet werden.

Die Skalp-Akupunktur, die einen Schwerpunkt des Projekts bildet, ist eine spezielle Art der Akupunktur, bei der Punkte am Kopf zur Behandlung verschiedener Krankheiten verwendet werden. Sie basiert auf der Theorie der 
Meridiane der TCM in Kombination mit der funktionellen Positionierung der Stimulation über der Großhirnrinde unter Verwendung relevanter Akupunkturpunkte am Kopf und der Aufteilung in mehrere entsprechende Stimulationsbereiche (Abb. 3).

Schlaflosigkeit ist eine häufige Erkrankung, bei der der Patient Probleme hat, durch- oder einzuschlafen. Insomnia ist $\mathrm{zu}$ einem globalen Gesundheitsproblem geworden. In der wissenschaftlichen Datenbank PubMed gibt es dazu mehr als 25.000 Rezensionen (Juli 2020). Einer dieser Artikel, der im Journal of Clinical Sleep Medicine [1] veröffentlicht wurde, weist auf die Notwendigkeit weiterer Untersuchungen im Zusammenhang zwischen den Auswirkungen der Akupunktur auf Schlaflosigkeit und der autonomen Regulation hin, die eine bessere selektive Anwendung dieser Behandlungsmethode bei Schlaflosigkeit ermöglichen könnten [1]. Die Ursachen für Schlaflosigkeit sind kompliziert. Einige Pathogene sind immer noch nicht klar. Die meisten Medikamente zur Behandlung von Insomnia haben nach längerer Anwendung Nebenwirkungen. Die Akupunktur-Therapie wurde in der klinischen Forschung gegen Schlaflosigkeit schrittweise angewendet, und ihre Wirksamkeit wurde allgemein anerkannt.

Die Herzratenvariabilität (HRV) ist ein objektiver Marker, der Einblick in die Dynamik des autonomen Nervensystems gibt. Die Beziehung zwischen sympathischem und parasympathischem Nervensystem ist antagonistisch und harmonisch, genau wie die Beziehung zwischen Yin und Yang in der TCM. Das veränderte Gleichgewicht des autonomen Nervensystems bei PatientInnen mit Schlaflosigkeit wurde von ForscherInnen nachgewiesen und es wurden Hinweise auf eine HRV-Beeinträchtigung bei SchlaflosigkeitspatientInnen gefunden [2].

Frühere Studien an PatientInnen mit Depressionen [2] und nach Schlaganfall [3] in Harbin, an PatientInnen mit Burnout [4] und tierexperimentelle Untersuchungen in Peking [5] haben gezeigt, dass das Konzept der transkontinentalen Tele-Akupunkturstudien gut funktioniert. Dies bedeutet, dass die Daten bei PatientInnen in China erfasst und in Österreich analysiert werden [2-7].

\section{Methode}

Ziel dieses Forschungsprojekts ist die Bewertung eines möglichen zusätzlichen klinischen Effekts der Skalp-Akupunktur zurVerbesserung des Schlafes und zurVeränderung der HRV bei PatientInnen mit Schlaflosigkeit.

Die klinische Pilotstudie mit beteiligter Grundlagenforschung in Graz wird an der Abteilung für Akupunktur und Moxibustion des zweiten angegliederten Kranken-

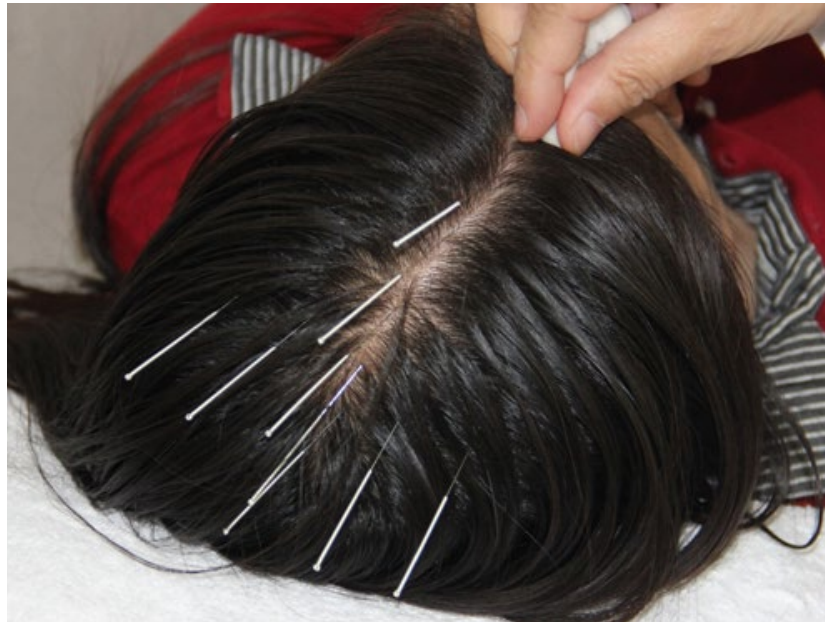

Abb. 3: Skalp-Akupunktur in Harbin, China

hauses der Heilongjiang-Universität für Chinesische Medizin und im TCM-Forschungszentrum der Medizinischen Universität Graz durchgeführt. 40 PatientInnen mit primärer Schlaflosigkeit werden imVerhältnis 1:1 in 2 Gruppen geteilt und randomisiert.

Die Einschlusskriterien lauten wie folgt:

- Sowohl nach der Chinesischen als auch nach der Westlichen Medizin wurde primäre Schlaflosigkeit diagnostiziert.

- Rekrutiert werden männliche und weibliche Patienten im Alter von 18-70 Jahren.

- Der Pittsburgh Sleep Quality Index (PSQI) beträgt mehr als 7 .

- Die PatientInnen haben seit 3 Monaten keine Behandlung gegen Schlaflosigkeit erhalten.

- Keine anderen Krankheiten beeinträchtigen den Schlaf.

- Die PatientInnen stimmen den Messungen zu, verstehen die Behandlung und unterzeichnen das Protokoll.

Die Ausschlusskriterien sind folgendermaßen festgelegt:

- PatientInnen erfüllen nicht die Diagnose einer primären Schlaflosigkeit.

- Schwangere.

- PatientInnen mit schwerer Grunderkrankung.

- PatientInnen, die Anti-Angst-, Antidepressiva-Medikamente oder Schlaftabletten verwenden.

\section{Interventionen und Bewertung}

In beiden Gruppen wird Akupunktur 5-mal pro Woche für 30 Minuten pro Behandlung für 2 Wochen verabreicht. Die Behandlungsgruppe erhält an den Punkten LG 20 (Baihui), LG 24 (Shenting) und Gb 13 (Benshen; bilateral) eine Skalp-Akupunktur. Auch einige allgemeine Körper- 
punkte für die Behandlung von Schlaflosigkeit, einschließlich EX-HN 3 (Yintang), EX 8 (Anmian I), KS 6 (Neiguan; bilateral), He 7 (Shenmen; bilateral), Bl 62 (Shenmai; bilateral) und Ni 6 (Zhaohai; bilateral), werden genadelt und stimuliert. Die Kontrollgruppe erhält nur allgemeine Körper-Akupunktur ohne Skalp-Akupunktur.

Die HRV wird vor und nach den gesamten Behandlungssitzungen sowie 5 Minuten vor bis 5 Minuten nach jeder Behandlung verglichen.

Die primären Evaluierungsparameter für die Ergebnisse der Studie sind die HRV-Indizes und die PSQI-Schlafskala, die sekundären Ergebnisse sind der Insomnia Severity Index und die HAMA (Hamilton-Angstskala) oder die HAMD (Hamilton-Depressionsskala).

\section{Konklusion}

Die gemeinsame Chinesisch-Österreichische Forschung im Rahmen dieses Projekts zielt auf die wissenschaftliche Bewertung von Schlaflosigkeit mittels Skalp- und Körperakupunktur ab. Als eine Beurteilungsgröße wird die HRV herangezogen. Dieser Parameter ist ein sehr guter Indikator für den Gesundheitszustand und kann durch verschiedene Akupunkturmethoden beeinflusst werden. Die Anzahl der zu untersuchenden PatientInnen und die Dauer des Projekts hängen auch von der kontinuierlichen

\section{Literatur}

[1] Huang W, Kutner N, Bliwise DL: Autonomic activation in insomnia: the case for acupuncture.J Clin Sleep Med 2011; 7: 95-102.

[2] Wang L, Cheng G, Sheng Z et al.: Clinical teleacupuncture between China and Austria using heart rate variability in patients with depression, Chinese Medicine 2011; 2: 71-76.

[3] Wang L,Valentini J, Sugimoto K et al.: Biomedical teleacupuncture between China and Austria using heart rate variability, part 1: poststroke patients. Evid Based Complement Alternat Med 2011;2011: 782489

[4] Litscher G: Modernization of traditional acupuncture using multimodal computer-based high-tech methods-recent results of blue laser and teleacupuncture from the Medical University of Graz. J Acupunct Meridian Stud 2009; 2: 202-209.
Weiterfinanzierung des Projekts in China ab. Erste Daten wurden bereits auch während der Corona-Krise in China erhoben und in Graz analysiert und zeigen, dass die Funktionsfähigkeit des Prozederes gegeben ist.

\section{Danksagung}

In Österreich wird die Arbeit vom Eurasia Pacific Uninet (EPU-Projekt 35/2019; ,Effekte der Skalp-Akupunktur auf die Herzratenvariabilität bei Patienten mit Schlafstörungen“; Projektleiter G. Litscher) unterstützt.

\section{Beteiligte ForscherInnen}

Univ.-Prof. DI DDr. Gerhard Litscher (Projektleiter, Medizinische Universität Graz, Gastprofessor an der Heilongjiang-Universität für TCM Harbin, China), Priv.-Doz. Dr. med. Lu Wang (Medizinische Universität Graz, Gastprofessorin an der Heilongjiang-Universität für TCM Harbin, China), Dr. Zemin Sheng (Privatklinik Lassnitzhöhe und Gastprofessor an der Heilongjiang-Universität für TCM Harbin, China), Prof. Yuanzheng Sun (Direktor, Second Affiliated Hospital der Heilongjiang-Universität fürTCM Harbin, China),Yan Yang und TianyangYu (beide Heilongjiang-Universität für TCM Harbin, China).

Interessenkonflikt: Die Autoren erklären keinen Interessenkonflikt.
[5] Gao XY, Liu K, Zhu B, Litscher G: Sino-European transcontinental basic and clinical high-tech acupuncture studies, part 1: Auricular acupuncture increases heart rate variability in anesthetized rats. Evid Based Complement Alternat Med 2012; 2012: 817378. doi:10.1155/2012/817378.

[6] Litscher G:Teleacupuncture: A new approach for transcontinental long-distance cooperation between Graz, Austria and Beijing, China (7,650 km). Medical Acupuncture 2009; 21: 223-224.

[7] Litscher G, ed.: Heart rate variability and acupuncture. Results from transcontinental studies. Lengerich: Pabst Science Publishers; 2016.

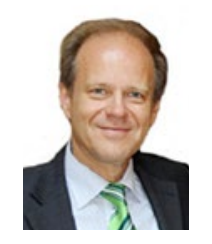

Univ.-Prof. DI DDr. Gerhard Litscher

Medizinische Universität Graz

Auenbruggerplatz 39, 8036 Graz

gerhard.litscher@medunigraz.at 